\title{
THE SPECTRA FOR OPERATORS OF A BASIC COLLECTION
}

BY R. K. JUBERG

Communicated by H. F. Weinberger, November 27, 1972

We present here the spectra of operators from a basic collection considered in the scale of Lebesgue spaces of $p$ th power summable functions over a finite interval.

Without loss of generality we confine our attention to complex valued functions over the interval $[0,1]$. The associated Banach spaces are denoted by $L^{p}, 1<p<\infty$. The results lift to any underlying bounded interval $[a, b]$ through, for example, the mappings induced by the linear $\operatorname{map}[a, b] \ni s \mapsto(b-a)^{-1}(s-a)=t \in[0,1]$.

The results unfold primarily through certain formal manipulations on some basic relations in an algebra of elementary operations.

On complex valued functions over the interval $[0,1]$ we consider the operations (see [2])

$$
\begin{aligned}
J^{\beta} \psi(t) & =\Gamma(\beta)^{-1} \int_{0}^{t}(t-x)^{\beta-1} \psi(x) d x, & & 0<\operatorname{Re} \beta, \\
& =\lim _{b \rightarrow 0+} J^{b+\beta} \psi(t) \quad\left(L^{p} \text {-limit }\right), & & \operatorname{Re} \beta=0, \\
& =d J^{\beta+1} \psi(t) / d t, & & -1<\operatorname{Re} \beta<0,
\end{aligned}
$$

and

$$
\begin{aligned}
J^{* \beta} \psi(t) & =\Gamma(\beta)^{-1} \int_{t}^{1}(x-t)^{\beta-1} \psi(x) d x, & & 0<\operatorname{Re} \beta, \\
& =\lim _{b \rightarrow 0+} J^{* b+\beta} \psi(t) \quad\left(L^{\left.p_{\text {-limit }}\right),}\right. & & \operatorname{Re} \beta=0, \\
& =-d J^{* \beta+1} \psi(t) / d t, & & -1<\operatorname{Re} \beta<0 .
\end{aligned}
$$

In addition let $M^{\gamma}$ denote the operation given by $M^{\gamma} \psi(t)=t^{\gamma} \psi(t), \gamma-$ complex, and $R$ the operation $R \psi(t)=\psi(1-t)$. Further denote by $H$ the finite Hilbert transform

$$
H \psi(t)=\frac{1}{\pi}(\text { p.v. }) \int_{0}^{1} \frac{\psi(x)}{t-x} d x,
$$

the integral being the Cauchy principal value. We consider also $H$ as

\footnotetext{
AMS (MOS) subject classifications (1970). Primary 47A10, 46E30, 26A33; Secondary 45E10.

Key words and phrases. Spectral properties, $L^{p}$ space, fractional integration/differentiation. Copyright (C) American Mathematical Society 1973
} 
extended to a bounded operator in $L^{p}, 1<p<\infty$.

The algebra of elementary operations mentioned above is that generated by the collection $\left\{J^{\beta}, R, M^{\gamma}\right\}$.

The basic operators under study here are those defined by the operations

$$
\psi \rightarrow J^{\alpha} J^{*-\alpha} \psi, \quad|\operatorname{Re} \alpha|<1 .
$$

Such operators arise quite naturally in diverse settings; see, for example, [3] and Kalisch [4].

The results to be presented here, combined with those in [2] and [3], lend themselves to the analysis of some fundamental operators; in particular, the classical operators defined for $0<\operatorname{Re} \alpha<1$ by

$$
S_{\alpha} \psi(t)=\int_{0}^{1} \frac{\psi(x)}{|t-x|^{1-\alpha}} d x, \quad T_{\alpha} \psi(t)=\int_{0}^{1} \frac{\operatorname{sgn}(t-x)}{|t-x|^{1-\alpha}} \psi(x) d x .
$$

Note that $\Gamma(\alpha)^{-1} S_{\alpha}=J^{\alpha}+J^{* \alpha}$ and $\Gamma(\alpha)^{-1} T_{\alpha}=J^{\alpha}-J^{* \alpha}$. Then on formally factoring one encounters the operations (1). Such study will be included elsewhere.

The question of when the operations (1) give rise to bounded operators in $L^{p}$ was considered in [3], and it is summarised here.

THEOREM 1. The operation $\psi \rightarrow J^{\alpha} J^{*-\alpha} \psi,|\operatorname{Re} \alpha|<1, \alpha \neq 0$, defines a bounded operator in $L^{p}, 1<p<\infty$, if and only if $-1<p \operatorname{Re} \alpha<p-1$.

A fundamental relation in the algebra is that expressed in the following (see [2], [3]).

TheOREM 2. For $|\operatorname{Re} \beta|<1$,

$$
(\cos \pi \beta) I+(\sin \pi \beta) H=M^{-\beta} J^{\beta} J^{*-\beta} M^{\beta} .
$$

With the aid of this basic relation (2) we are led to the following.

TheOREM 3. For $z=\sin \pi \alpha \cot \pi \zeta+\cos \pi \alpha,|\operatorname{Re} \alpha|<1,|\operatorname{Re} \zeta|<1$, $\zeta \neq 0$

$$
\begin{aligned}
& \left(M^{\alpha+\zeta} R M^{-\alpha-\zeta}\right) J^{\alpha} J^{*-\alpha}\left(M^{\alpha+\zeta} R M^{-\alpha-\zeta}\right) \\
& \quad=(\cos \pi \alpha) I+(\sin \pi \alpha)\left(M^{\alpha+\zeta} R M^{-\zeta}\right) H\left(M^{\zeta} R M^{-\alpha-\zeta}\right)
\end{aligned}
$$

and

$$
\begin{gathered}
(\sin \pi \zeta)^{2}\left(M^{\alpha+\zeta} R M^{-\alpha-\zeta}\right)\left(z I-J^{\alpha} J^{*-\alpha}\right)\left(M^{\alpha+\zeta} R M^{-\alpha-\zeta}\right)\left(z I-J^{\alpha} J^{*-\alpha}\right) \\
=(\sin \pi \alpha)^{2} I .
\end{gathered}
$$

The equalities (3) and (4) are valid for $\operatorname{Re} \alpha \neq 0$ as arithmetical identities on applying each side to an arbitrary smooth function compactly sup- 
ported in $(0,1)$ and in the sense of $L^{p}(1<p<\infty)$ when $\operatorname{Re} \alpha=0$.

TheOREM 4. For $|\operatorname{Re} a|<1,|\operatorname{Re} b|<1$ the operation

$$
\psi \rightarrow\left(M^{a} R M^{b}\right) H\left(M^{-b} R M^{-a}\right) \psi
$$

defines a bounded operator in $L^{p}, 1<p<\infty$, if and only if, $-1<p \operatorname{Re} a$ $<p-1$ and $-1<p \operatorname{Re} b<p-1$.

The special case of Theorem 4 where $a=0$ played a central role in the proof of Theorem 1 ; see [3]. In addition, the case where $\operatorname{Re}(a+b)=0$ reduces to Theorem 3 in [2].

The above theorems combine to give the following.

TheOREM 5. Let $|\operatorname{Re} \alpha|<1, \alpha \neq 0$, and $1<p<\infty$. Denote by $K_{\alpha}$ the operator defined in $L^{p}$ by $J^{\alpha} J^{*-\alpha}$ where $-1<p \operatorname{Re} \alpha<p-1$.

(i) Spectrum

$$
\begin{array}{r}
\operatorname{sp}\left(K_{\alpha} \mid L^{p}\right)=\mathrm{cl}-\left\{\sin \pi \alpha \cot \pi \zeta+\cos \pi \alpha:\left|\operatorname{Re} \zeta-\frac{1}{2}(1-\operatorname{Re} \alpha)\right|\right. \\
\left.\leqq\left|\frac{1}{2}(1-\operatorname{Re} \alpha)-p^{-1}\right|\right\} .
\end{array}
$$

(ii) The resolvent set

$$
\begin{array}{r}
\rho\left(K_{\alpha} \mid L^{p}\right)=\left\{\sin \pi \alpha \cot \pi \zeta+\cos \pi \alpha:\left|\operatorname{Re} \zeta+\frac{1}{2} \operatorname{Re} \alpha\right|\right. \\
\left.<\frac{1}{2}-\left|\frac{1}{2}(1-\operatorname{Re} \alpha)-p^{-1}\right|, \zeta \neq 0\right\}
\end{array}
$$

and for $z \in \rho\left(K_{\alpha} \mid L^{p}\right)$ the resolvent

$\left(z I-K_{\alpha}\right)^{-1}=(\csc \pi \alpha)^{2}(\sin \pi \zeta)^{2}\left(M^{\alpha+\zeta} R M^{-\alpha-\zeta}\right)\left(z I-K_{\alpha}\right)\left(M^{\alpha+\zeta} R M^{-\alpha-\zeta}\right)$

where $z=\sin \pi \alpha \cot \pi \zeta+\cos \pi \alpha$.

REMARKS. (I) In the case $p(1-\operatorname{Re} \alpha)=2$ the spectrum is the circular arc with endpoints $e^{ \pm i \pi \alpha}$ that contains the point 1 . The limiting, or degenerate, case where $\operatorname{Re} \alpha=0$ is an interval on the real axis.

(II) For a case where $p=2$ the spectrum is the segment of the disk bounded by the circle through the points 0 and $e^{ \pm i \pi \alpha}$ that is cut off by the secant with endpoints $e^{ \pm i \pi \alpha}$ and that contains the point 1 . As in (I) the limiting case where $\operatorname{Re} \alpha=0$ is again the interval on the real axis.

(III) The cases where $\alpha=i \tau$ (that is, $\operatorname{Re} \alpha=0$ ) relate to the continuous boundary group of the holomorphic semigroup (see Hille-Phillips $[1, \S 23.16])$. Here the spectrum for each $\tau$ is described using the disks bounded by the circles centered at $c$ and $\bar{c}$,

$$
c=\cosh \pi \tau+i(\sinh \pi|\tau|)|\cot 2 \pi / p|,
$$

that pass through the points $e^{ \pm \pi|\tau|}$.

(a) For $0<\left|p^{-1}-\frac{1}{2}\right| \leqq \frac{1}{4}$ the spectrum is the intersection of the disks. 
(b) For $p=2$ the spectrum is the interval $\left[e^{-\pi|\tau|}, e^{\pi|\tau|}\right]$.

(c) For $\left|p^{-1}-\frac{1}{2}\right| \geqq \frac{1}{4}$ the spectrum is the union of the disks.

This is similar to the situation for the finite Hilbert transform given in [2].

\section{REFERENCES}

1. E. Hille and R. S. Phillips, Functional analysis and semi-groups, Amer. Math. Soc. Colloq. Publ., vol. 31, Amer. Math. Soc., Providence, R.I., 1965.

2. R. K. Juberg, Finite Hilbert transforms in $L^{p}$, Bull. Amer. Math. Soc. 78 (1972), $435-438$.

3. - On the boundedness of certain singular integral operators, Colloq. Math. Soc. Janos Bolyai 5, Hilbert space operators, Tihany, Hungary, 1970.

4. G. K. Kalisch, On the similarity of certain operators, ibid.

Department of Mathematics, University of California, Irvine, California 92664

Current address: Department of Mathematics, University of Sussex, Falmer, Brighton (BN1 9QF), England 